\title{
Le concours pour arrêter de fumer favorise la désaccoutumance au tabac
}

\author{
Une recherche confirme à nouveau qu'il vaut la peine d'arrêter de fumer à tout âge. \\ Le concours pour arrêter de fumer organisé pour la Journée mondiale sans tabac du \\ 31 mai est une occasion idéale de motiver les fumeuses et les fumeurs à arrêter.
}

Abhinay Agarwal

Responsable de projet

Correspondance: Abhinay Agarwal Association suisse pour la prévention du tabagisme Haslerstrasse 30

CH-3008 Berne

Tél. 0315991020

Fax 0315991035

info@at-schweiz.ch
Le tabagisme accélère aussi la diminution des fonctions cognitives liées à l'âge. Une équipe de recherche de l'Université d'Australie-Occidentale a analysé l'effet du tabagisme sur les capacités cognitives de 332 personnes d'un âge mûr. Après vingt-quatre mois sans fumer, leurs fonctions cognitives étaient à nouveau identiques à celles des jamais-fumeurs [1].

«Du point de vue de la santé, un résultat est particulièrement important: les fumeuses et fumeurs chroniques qui arrêtent de fumer présentent un déficit cognitif moins important et de meilleures capacités intellectuelles que celles et ceux qui ont continué à fumer», a expliqué le responsable de la recherche Osvaldo Almeida.

\section{Pause tabac}

Le concours pour arrêter de fumer implique d'arrêter de fumer entre le 4 juin et le 4 juillet 2011. Cette pause tabac de quatre semaines augmente les chances d'arriver à se débarrasser définitivement de sa dépendance à la nicotine. Les prix tirés au sort sont de $1 \times 5000$ francs et de $10 \times 500$ francs. L'assurance-maladie Assura met également en jeu une semaine de vacances pour deux personnes.

Les fumeuses et les fumeurs peuvent s'inscrire en ligne sur www.at-suisse.ch ou par écrit. Des cartes d'inscription peuvent être obtenues gratuitement auprès de l'Association suisse pour la prévention du tabagisme sur www.at-suisse.ch ou au 0315991020.

\section{Enquête représentative}

L'Institut LINK de recherche marketing et sociale à Lucerne et le Bureau d'études et de communication Hans Krebs à Zurich ont interrogé en janvier 2010 un échantillon transversal de 505 participants et participantes au concours 2009 sur mandat de l'Association suisse pour la prévention du tabagisme:

- 64 pour cent ont réussi à rester un mois sans fumer. 33 pour cent n'avaient pas recommencé à fumer six mois après le concours. Ceux qui avaient recommencé fumaient nettement moins de cigarettes qu'avant le concours.

- 62 pour cent des personnes interrogées ont utilisé une ou plusieurs aides à la désaccoutumance au tabac, surtout des médicaments: 25 pour cent ont recouru aux substituts nicotiniques, 6 pour cent à la varénicline et 2 pour cent au buproprion.
Ouvrages, brochures, dépliants d'informations ont aussi été très demandés.

\section{FMH partenaire}

Les organisations partenaires du concours pour arrêter de fumer sont la Fédération des médecins suisses, pharmaSuisse, la Société suisse d'odonto-stomatologie, l'Association suisse des infirmières et infirmiers et Swiss Dental Hygienists.

Le concours est une action du programme national d'arrêt du tabagisme pour la Journée mondiale sans tabac du 31 mai. Les organismes responsables sont la Ligue suisse contre le cancer, la Fondation suisse de cardiologie et l'Association suisse pour la prévention du tabagisme. Le fonds de prévention du tabagisme apporte son soutien financier au concours. Les ligues cantonales contre le cancer, les ligues pulmonaires cantonales, les centres de prévention et d'autres organismes de prévention et de promotion de la santé sont responsables de sa mise en œuvre.

\section{Référence}

1 Osvaldo P. Almeida et al. 24-Month effect of smoking cessation on cognitive function and brain structure in later life. Dans: NeuroImage Article in Press doi:10.1016/j.neuroimage.2011.01.063 www.sciencedirect.com/science/journal/10538119

\section{Ligne stop-tabac 0848000181}

Si le temps manque pour un entretien détaillé, les fumeuses et les fumeurs peuvent être renvoyés à la ligne nationale stop-tabac. Les conseillères et les conseillers sont dûment formés et connaissent les diverses méthodes pour arrêter de fumer qui ont fait leurs preuves au plan scientifique. Ils peuvent accompagner les fumeuses et fumeurs intéressés pendant leur sevrage tabagique et les rappeler jusqu'à quatre fois.

La ligne stop-tabac 0848000181 est ouverte de 11 heures à 19 heures du lundi au vendredi pour des conseils en français, en allemand ou en italien (8 centimes la minute sur le réseau fixe). Un entretien spécialisé en albanais, en portugais, en serbe-croate-bosniaque, en espagnol et en turc est également possible, avec un numéro de téléphone pour chacune de ces langues, mentionné sur la carte d'inscription. 